\title{
Pulsar Temporal Studies with SPI
}

\author{
L. Borgonovo*, S. Molkov, E. Jordain, and J.-P. Roques \\ Centre d'Etude Spatiale des Rayonnements (CESR) \\ E-mail: luis-maria.borgonovo@cesr.fr
}

The good timing capabilities of the spectrometer SPI on INTEGRAL makes it a suitable tool for temporal studies although so far this quality has not been fully exploited. Preliminary results of the time analysis of SPI event data from the bright accreting pulsars EXO 2030+375 and A $0535+262$ are shown, illustrating the instrument's potential for those purposes. Complemented with IBIS/ISGRI and other X-ray instruments, SPI allows a broad energy band coverage which is particularly relevant for pulsar studies, since comparative multi-wavelength analysis of pulse profiles are essential to understand their emission mechanism. We derived the pulse profiles and used hardness ratios to analyze the pulse spectral evolution.

7th INTEGRAL Workshop

September 8-11 2008

Copenhagen, Denmark

\footnotetext{
* Speaker.
} 


\section{Introduction}

The SPI spectrometer on board INTEGRAL has good timing capabilities that makes it suitable for temporal studies. These have been demonstrated, e.g., by Kuiper et al. (2003) who have estimated an absolute arrival time accuracy of $130 \mu$ s based on analysis of Crab pulsar observations. This high accuracy is particularly important when long integration times are needed. Thanks to the mission routine survey of the galactic plane, many high-energy sources of interest are regularly covered by INTEGRAL observations over a long time span. Aside from the particular energy range covered by the combined IBIS/SPI instruments, from the temporal study point of view the long accumulated observation time on those sources gives unique benefits over other currently operating high-energy observatories with higher sensitivity or timing accuracy. However, to allow for proper image deconvolution of the SPI mask, the standard data reduction software provided by the INTEGRAL Science Data Centre (ISDC) requires a minimum data set unit of one science window, which implies a temporal resolution for the reduced data no better than $\sim 30 \mathrm{~min}$, a serious limitation for most temporal studies.

Since SPI data is dominated by background noise even for the brightest sky sources, there is a considerable trade-off between signal-to-noise ratio $(\mathrm{S} / \mathrm{N})$ and time resolution for using the full event data undeconvolved. However, taking into account the shadow pattern of the mask on the array of detectors and selecting the ones with larger illumination fractions from the source, a compromise solution can be reached. In the following sections we report on the implementation of this procedure for the SPI instrument and we show preliminary results on the study of pulse profiles of two bright pulsars illustrating the potentiality of the instrument for this kind of analysis.

\section{Methods}

Since we want to retain the full temporal resolution of the instrument, we make use of the mask in the crudest way. If a single source is present in the field of view, then detectors that are poorly illuminated are simply disregarded (e.g., for this work we chose empirically a threshold of $50 \%$ illumination fraction). For an on-axis source this represents about a factor two gain in $\mathrm{S} / \mathrm{N}$, however if the source is off-centered the improvement can be better than an order of magnitude. Figure 1 shows the mask shadow pattern on the SPI detectors for a case were there are three bright sources in the field of view. As illustrated in panel (b), in principle the signal from a particular source could be separated from the rest by choosing the detectors that are exclusively Illuminated by it. In practice there may be none and a fraction of "contamination" from the other sources may have to be tolerated. In this context, sources emitting coherent signals like pulsars are ideal for analysis since they can be treated as isolated sources. By using standard epoch folding techniques, the contribution from other sources have a random uniform distribution over the pulse phase that becomes part of the general background noise flat level.

\section{Pulsar Temporal Analysis}

The Be/X-ray binary systems are the most common type of accreting X-ray pulsar systems. They consist of a compact object and a main sequence star of type B that presents Balmer lines 
a)

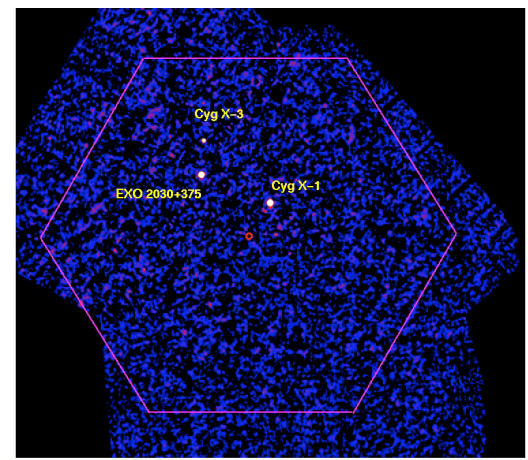

b)

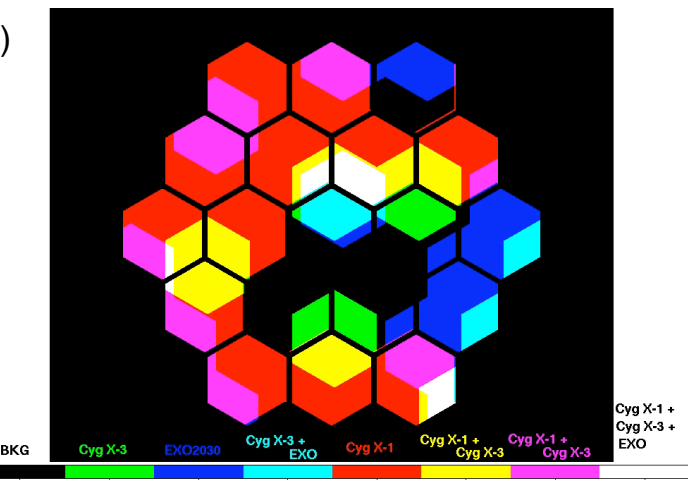

Figure 1: a) SPI image showing the field of view of pulsar EXO2030+375, where two additional bright sources are present within the full coded hexagonal area, i.e., the X-ray binaries Cyg X-1 and Cyg X-3. b) Sketch illustrating the illumination pattern over the 19 SPI detectors. The color code indicates which sources illuminate each detector.
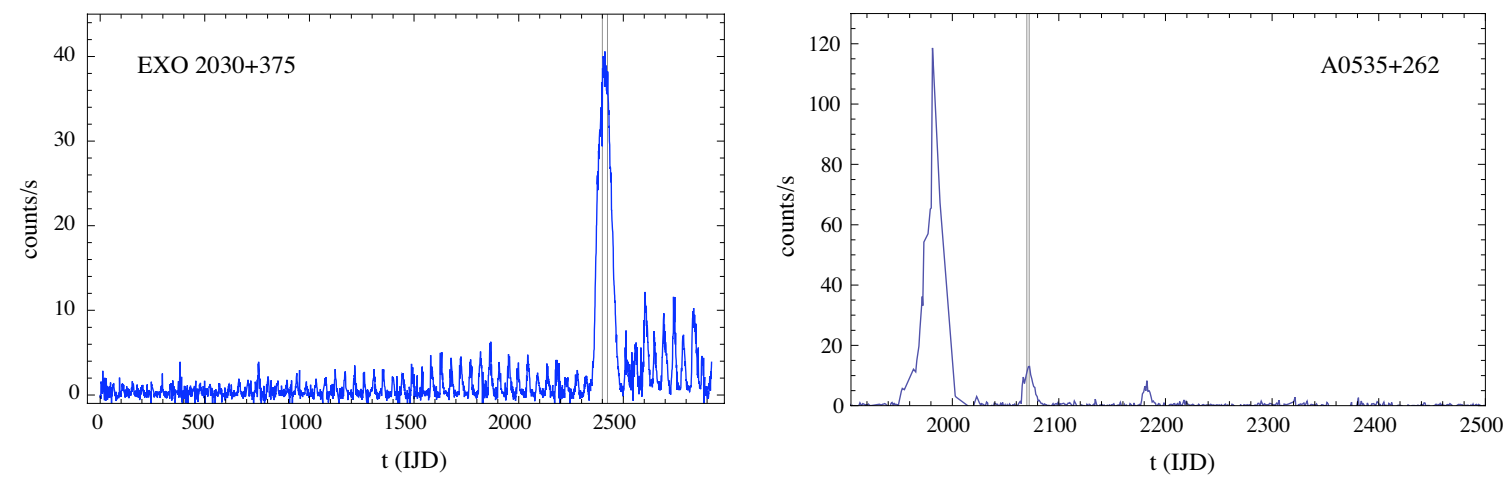

Figure 2: ASM data from EXO 2030+375 and A 0535+262. The vertical lines indicate the time of INTEGRAL observations analyzed in this work (IJD $\equiv$ MJD-51544 days).

in emission. The general believe is that this is due to a disk-like equatorial outflow created by the fast spinning of the star. The accreting pulsar shows regular outbursts that concur with the orbital periastron passage when the strongest interactions with the star occur. The outburst are classified into two types: the type I is the most common, it reaches moderate luminosities, and little or none increase in the pulsar spin rate is observed associated with it; the type II or giant outburst is characterized by much higher luminosities followed by a continuous increase in the spin frequency of the pulsar even many orbital periods after the event. This suggests that during this second type of outburst an accretion disk is formed around the pulsar that produces the increase in angular momentum (Coe 2000), the existence of which is further supported by the occasional detection of quasi-periodic oscillations (QPOs) during these transients (Angelini et al. 1989).

\subsection{Pulsar EXO 2030+375}

EXO 2030+375 is a well-studied Be/X-ray binary (see, e.g., Wilson et al. 2002) that was discovered in 1985 by EXOSAT during a giant outburst when it reached crab X-ray luminosities 

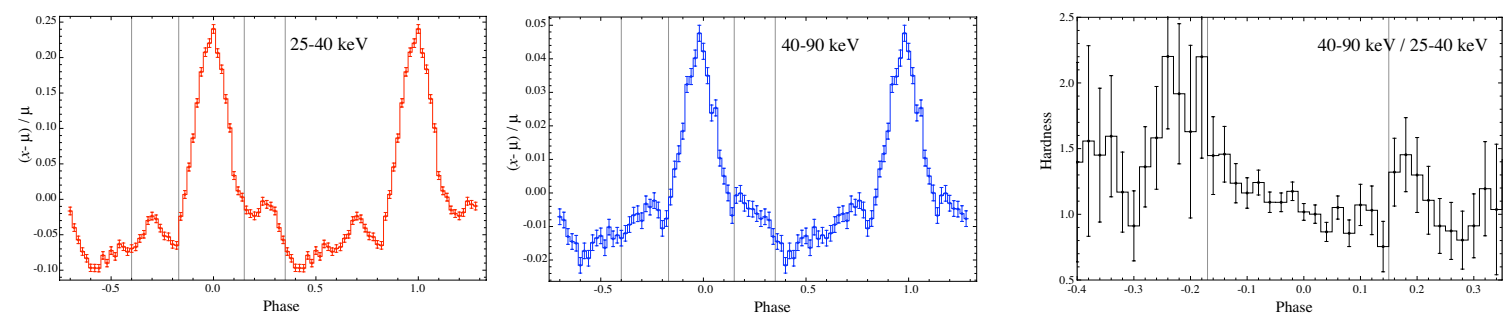

Figure 3: EXO 2030+375 pulse profile at two X-ray energy bands. On the right, the hardness ratios derived from them.

(Parmar et al. 1985). INTEGRAL was able to make target observations at the peak of its most recent giant outburst that occurred in 2006, as indicated in Figure 2 where we show the activity of the X-ray source as followed daily by the All Sky Monitor (ASM) onboard the Rossi X-ray Timing Explorer (RXTE). Regular type I outbursts are clearly seen at even time intervals of $P_{\text {orb }} \approx 46$ days, the orbital period of the binary. The spin period of the pulsar is $P_{\text {spin }} \approx 42 \mathrm{~s}$.

SPI event data was selected following the method described above. Times were translated to the solar system barycenter and the orbital motion of the binary was corrected using orbital parameters from Wilson et al. (2002). Pulse profiles obtained using epoch folding techniques are shown in Figure 3. The energy range where substantial emission was detected was split in two bands having similar S/N levels in order to obtain good hardness estimates. The pulse profile could be roughly described as having a main peak with "side-lobes". We see in the third panel that the phase spectral evolution during these phase intervals is clearly different, displaying a monotonic hard-to-soft change over the main peak. Some pulsed emission is observed at higher energies, but at a marginal level of detection with $3.7 \sigma_{\mathrm{N}}\left(\chi^{2}\right.$-test) in the 90-400 keV energy band.

EXO $2030+375$ pulse profiles were previously obtained by Klochkov et al. (2007) corresponding to the same giant outburst and covering similar energy range, but using data from the IBIS/ISGRI experiment also onboard INTEGRAL. The profiles are fully consistent, although we do not resolve some small features due to the compromise in phase resolution imposed by the lower SPI sensitivity at these energies.

\subsection{Pulsar A 0535+262}

A $0535+262$ is another well-known accreting pulsar belonging to the subclass of Be/X-ray binaries. The source was discovered in 1975 by Ariel $V$ during a giant outburst (Coe et al. 1975) and it has been subject to numerous studies ever since (see, e.g., Giovannelli \& Graziati 1992). It has an orbital period $P_{\text {orb }} \approx 110$ days and a spin period $P_{\text {spin }} \approx 103$ s. In 1995 the X-ray source entered a long quiescent period until 2005 when another giant burst occurred, followed by regular type I events as can be seen in Figure 2. INTEGRAL did target observations of these last bursts, although unfortunately at the time of the peak of the main event the source was too close to the Sun to be observable.

We processed the corresponding SPI data as before, with the orbital motion of the binary corrected using orbital parameters estimated by Finger et al. (1996). The signal in this case allows us to obtain good profiles within the same energy range subdividing it in three bands as displayed in 

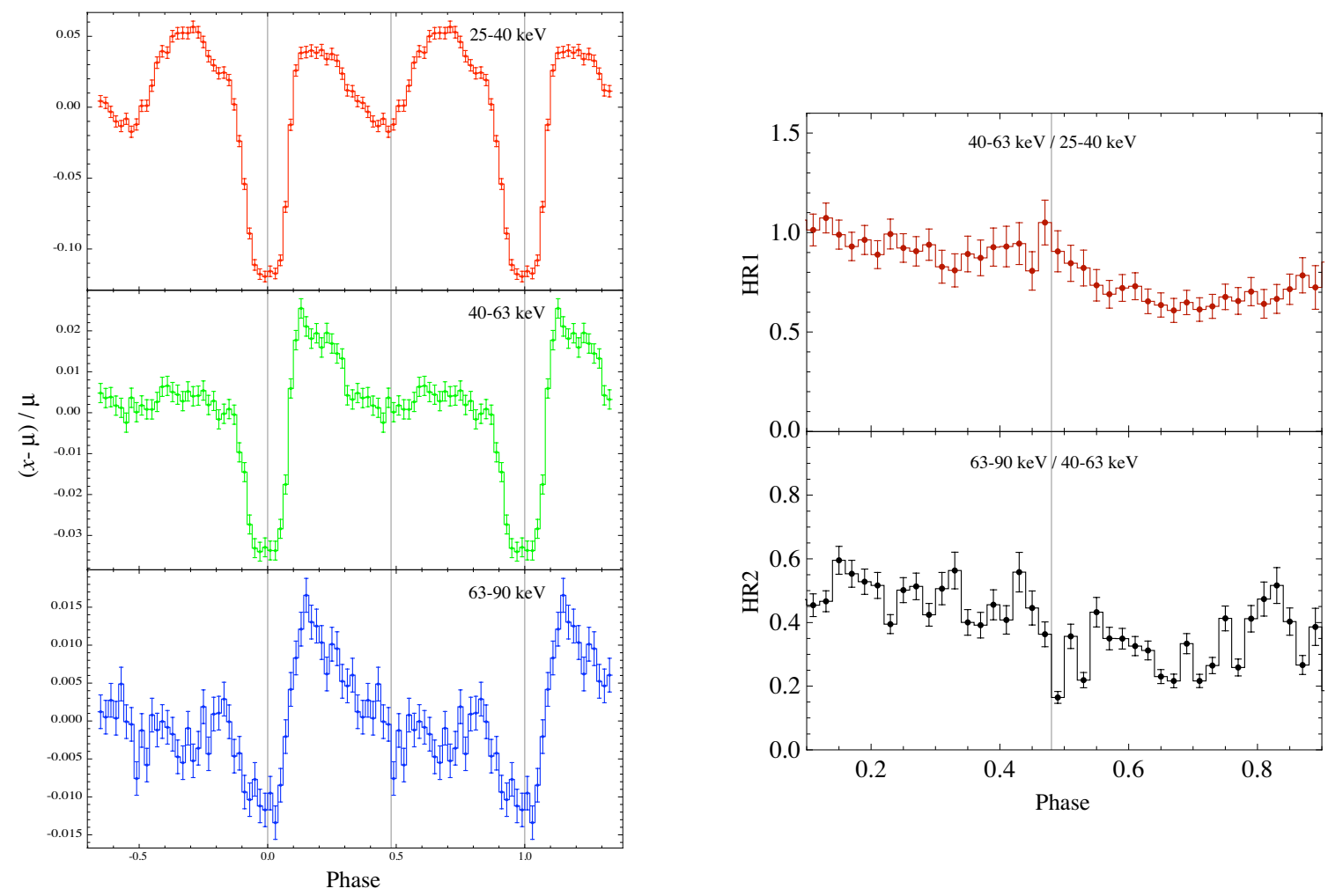

Figure 4: A $0535+262$ pulse profile at three energy bands (left), and the hardness ratios derived from them $($ right).

Figure 4. At the lowest energy band, the profile can be described as the overlap of two sub-pulses of similar intensity. However, the second component is softer, exhibits a much steeper spectral decline, and has a stronger phase dependence than the first. These results are in good agreement with pulse profiles reported by Caballero et al. (2008) based on the IBIS/ISGRI simultaneous observations. However, with SPI we were able to extend the analysis to much higher energies, detecting pulse emission at $4.9 \sigma_{\mathrm{N}}$ level $\left(\chi^{2}\right.$-test) for the $0.1-1 \mathrm{MeV}$ energy band and at $3.9 \sigma_{\mathrm{N}}$ level between $1-8 \mathrm{MeV}$.

\section{References}

Angelini, L., Stella, L., \& Parmar, A. N. 1989, ApJ, 346, 906

Caballero, I., Kretschmar, P., Santangelo, A., Segreto, A., Ferrigno, C., \& Staubert, R. 2008, LàrXivi:0801.389T!

Coe, M. J., Carpenter, G. F., Engel, A. R., \& Quenby, J. J. 1975, Nature, 256, 630

Coe, M. J. 2000, IAU Colloq. 175: The Be Phenomenon in Early-Type Stars, 214, 656

Finger, M. H., Wilson, R. B., \& Harmon, B. A. 1996, ApJ, 459, 288 
Giovannelli, F., \& Graziati, L. S. 1992, Space Science Reviews, 59, 1

Klochkov, D., et al. 2007, A\&A, 464, L45

Kuiper, L., Hermsen, W., Walter, R., \& Foschini, L. 2003, A\&A, 411, L31

Parmar, A. N., Stella, L., Ferri, P., \& White, N. E. 1985, IAU Circ., 4066, 1

Walter, R., et al. 2003, A\&A, 411, L25

Wilson, C. A., Finger, M. H., Coe, M. J., Laycock, S., \& Fabregat, J. 2002, ApJ, 570, 287 